NASA Contractor Report 202311

ICOMP-96-11

\title{
On Increasing the Accuracy of MacCormack Schemes for Aeroacoustic Applications
}

R. Hixon

Institute for Computational Mechanics in Propulsion

Cleveland, Ohio

December 1996

Prepared for

Lewis Research Center

Under Cooperative Agreement NCC3-483

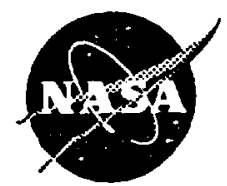

National Aeronautics and

Space Administration

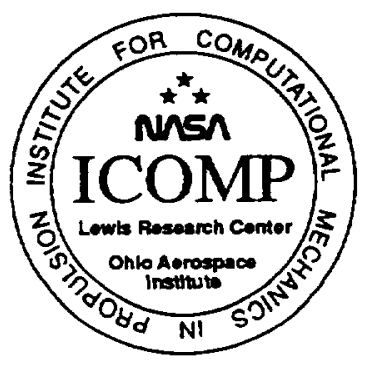


• 


\title{
On Increasing the Accuracy of MacCormack Schemes for Aeroacoustic Applications
}

\author{
R. Hixon \\ Institute for Computational Mechanics in Propulsion \\ Cleveland, Ohio 44135
}

\begin{abstract}
$\underline{\text { Abstract }}$
Due to their inherent dissipation and stability, the MacCormack scheme and its variants have been widely used in the computation of unsteady flow and acoustic problems. However, these schemes require many points per wavelength in order to propagate waves with a reasonable amount of accuracy. In this work, the linear wave propagation characteristics of MacCormack-type schemes are investigated, and methods for greatly improving their performance are described and demonstrated.
\end{abstract}

\section{Introduction}

In the field of computational aeroacoustics, numerical schemes are expected to propagate waves accurately for long distances over long periods of time. In order to accomplish this goal, a certain number of spatial points are required per wavelength to model each wave, and a certain time step is required in order to model the wave's movement in time. It is desirable from a computational standpoint to reduce the number of points required per wavelength and increase the size of the allowable time step. 
One popular and well-tested method uses a modification of the MacCormack scheme [1], which is second order accurate in time and fourth order accurate in space. This extension of the MacCormack scheme is known as the 2-4 scheme, and was described by Gottlieb and Turkel [2]. This scheme has been used successfully on a wide range of fluid and aeroacoustics problems [3-15]. Sankar, Reddy, and Hariharan have evaluated this scheme for aeroacoustics applications [16]. It has been extended to sixth- order spatial accuracy by Bayliss, et. al. (2-6 scheme) [17].

In this paper, these MacCormack-type schemes will be investigated in detail, and extensions to high-order accuracy will be developed. A test problem is used to quantify the performance of the various schemes. These schemes have been validated on the real-world problem of noise radiated by a supersonic jet using the linearized Euler equations.

\section{Test Equation and Numerical Formulation}

For comparative purposes, a simple hyperbolic equation is solved:

$$
\frac{\partial U}{\partial t}=-c \frac{\partial U}{\partial x}
$$

The harmonic solution to this equation is:

$$
U(x, t)=e^{i(k x-\omega x)}
$$

where $\mathrm{k}$ is the wavenumber and $\omega$ is the frequency. Here,

$$
w=c k \text {. }
$$

In order to numerically solve Eq. (1), the equation must be discretized in time and space. 
The discretized spatial derivatives must model accurately the derivative of the waveform:

$$
\frac{\partial U}{\partial x}=i k e^{i(k x-\omega x)}=\frac{1}{\Delta x} \sum_{j=-N}^{M} a_{j} U_{j}
$$

where the coefficients $a_{j}$ are the coefficients used in taking the spatial derivative.

Since:

$$
\begin{aligned}
U_{j} & =e^{i(k(x+j \Delta x)-\omega x)} \\
& =e^{i k j \Delta x} e^{i(k x-\omega t)}
\end{aligned}
$$

a numerical wavenumber can be defined, following Tam and Webb's work [18]:

$$
k^{*}=\frac{-i}{\Delta x} \sum_{j=-N}^{M} a_{j} e^{i k j \Delta x}
$$

In this way, Eq. (4) becomes:

$$
\frac{\partial U}{\partial x}=i k^{*} e^{i(k x-a x)}
$$

As the numerical scheme marches in time, the time integration must also model accurately the evolution of the waveform. From before:

$$
\begin{aligned}
\frac{\partial U}{\partial t} & =-c \frac{\partial U}{\partial x} \\
& =-i c k e^{i(k x-\omega x)} \\
& =-i \omega e^{i(k x-\omega x)}
\end{aligned}
$$

Integrating Eq. (8) gives:

$$
\begin{aligned}
U(x, t+\Delta t) & =e^{-i(\omega \Delta t)} e^{i(k x-\omega x)} \\
& =U(x, t) e^{-i(\omega \Delta t)}
\end{aligned}
$$

One popular method for numerical time integration is the Runge-Kutta scheme. A generic 
six-stage Runge-Kutta method has this form:

$$
\begin{aligned}
& U^{(1)}=U(x, t) \\
& U^{(2)}=U(x, t)-\alpha_{2} c \Delta t \frac{\partial U^{(1)}}{\partial x} \\
& U^{(3)}=U(x, t)-\alpha_{3} c \Delta t \frac{\partial U^{(2)}}{\partial x} \\
& U^{(4)}=U(x, t)-\alpha_{4} c \Delta t \frac{\partial U^{(3)}}{\partial x} \\
& U^{(5)}=U(x, t)-\alpha_{5} c \Delta t \frac{\partial U^{(4)}}{\partial x} \\
& U^{(6)}=U(x, t)-\alpha_{6} c \Delta t \frac{\partial U^{(5)}}{\partial x} \\
& \left.U(x, t+\Delta t)=U(x, t)-c \Delta t\left[\beta_{1} \frac{\partial U^{(1)}}{\partial x}+\beta_{2} \frac{\partial U^{(2)}}{\partial x}+\beta_{3} \frac{\partial U^{(3)}}{\partial x}\right]+\beta_{4} \frac{\partial U^{(4)}}{\partial x}+\beta_{5} \frac{\partial U^{(5)}}{\partial x}+\beta_{6} \frac{\partial U^{(6)}}{\partial x}\right]
\end{aligned}
$$

Using the coefficients from Table I for the sixth-order accurate Runge-Kutta scheme and using Eq. (7) to define the numerical wavenumber, Eq. (10) becomes:

$$
\begin{aligned}
U(x, t+\Delta t) & =U(x, t)\left[\begin{array}{l}
1-i c k^{*} \Delta t+\frac{\left(-i c k^{*} \Delta t\right)^{2}}{2}+\frac{\left(-i c k^{*} \Delta t\right)^{3}}{6} \\
+\frac{\left(-i c k^{*} \Delta t\right)^{4}}{24}+\frac{\left(-i c k^{*} \Delta t\right)^{5}}{120}+\frac{\left(-i c k^{*} \Delta t\right)^{6}}{720}
\end{array}\right] \\
& =U(x, t) e^{-i \omega^{*} \Delta t}
\end{aligned}
$$

Comparing Eq. (11) to Eq. (9), we can define the numerical frequency $\omega^{*}$ as:

$$
\omega^{*}=\frac{-i}{\Delta t} \ln \left[\frac{U(x, t+\Delta t)}{U(x, t)}\right]
$$




\section{Description of the MacCormack Scheme}

In the MacCormack scheme, integration in time is accomplished by applying an operator to the solution at the last time level. The time integration method used in the existing schemes is a second-order accurate Runge-Kutta method:

$$
\begin{aligned}
& U^{(1)}=U(x, t) \\
& U^{(2)}=U(x, t)-c \Delta t \frac{\partial U^{(1)}}{\partial x} \\
& U(x, t+\Delta t)=U(x, t)-c \frac{\Delta t}{2}\left[\frac{\partial U^{(1)}}{\partial x}+\frac{\partial U^{(2)}}{\partial x}\right]
\end{aligned}
$$

In the MacCormack scheme, one-sided differences are used in order to add dissipation to stabilize the scheme. The one-sided differences are defined in this way:

$$
\begin{aligned}
& \left.\frac{\partial U}{\partial x}\right|_{i} ^{F}=\sum_{j=-n}^{M} a_{j} U_{i+j} \\
& \left.\frac{\partial U}{\partial x}\right|_{i} ^{B}=\sum_{j=-n}^{M}-a_{j} U_{i-j}
\end{aligned}
$$

such that the underlying central difference is recovered when the forward and backward differences are added together:

$$
\begin{aligned}
& \left.\frac{\partial U}{\partial x}\right|_{i} ^{C}=\sum_{j=-M}^{M} A_{j} U_{i+j} \\
& A_{j}=\frac{1}{2}\left(a_{j}+a_{-j}\right)
\end{aligned}
$$

For example, in the original MacCormack scheme, forward and backward differences are 
defined as first-order accurate differences:

$$
\begin{aligned}
\left.\frac{\partial U}{\partial x}\right|_{i} ^{F} & =\frac{1}{\Delta x}\left(-U_{i}+U_{i+1}\right) \\
& =\frac{\partial U}{\partial x}+\Delta x \frac{\partial^{2} U}{\partial x^{2}}+\vartheta\left(\Delta x^{2}\right) \\
\left.\frac{\partial U}{\partial x}\right|_{i} ^{B} & =\frac{1}{\Delta x}\left(U_{i}-U_{i-1}\right) \\
& =\frac{\partial U}{\partial x}-\Delta x \frac{\partial^{2} U}{\partial x^{2}}+\vartheta\left(\Delta x^{2}\right)
\end{aligned}
$$

It is seen that adding (16) and (17) together recovers a second-order accurate central difference. In all of the existing MacCormack-type schemes, the one-sided differences are first-order accurate, with a dissipative leading error.

The forward and backward differences are alternately used in the time-stepping routine. For example, Eq. (13) becomes:

$$
\begin{aligned}
& U^{(1)}=U(x, t) \\
& U^{(2)}=U(x, t)-\left.c \Delta t \frac{\partial U^{(1)}}{\partial x}\right|^{F} \\
& U(x, t+\Delta t)=U(x, t)-c \frac{\Delta t}{2}\left[\left.\frac{\partial U^{(1)}}{\partial x}\right|^{F}+\left.\frac{\partial U^{(2)}}{\partial x}\right|^{B}\right]
\end{aligned}
$$




\section{Analysis of the MacCormack Scheme}

Using Eq. (6), the numerical wavenumbers can be found for the one-sided differences as well as the underlying central difference:

$$
\begin{aligned}
& k_{F}^{*}=\frac{-i}{\Delta x}\left(e^{i k \Delta x}-1\right)=\left(k^{*}+i \delta\right) \\
& k_{B}^{*}=\frac{-i}{\Delta x}\left(-e^{-i k \Delta x}+1\right)=\left(k^{*}-i \delta\right) \\
& k_{C}^{*}=\frac{-i}{\Delta x}\left(e^{i k \Delta x}-e^{-i k \Delta x}\right)=k^{*}
\end{aligned}
$$

Notice that the real part of the numerical wavenumber is identical to that of the underlying central difference for all differences, and that the imaginary parts are due to the form of the one-sided differences and are equal and opposite.

Putting these definitions into the time integration method gives:

$$
\begin{aligned}
U^{(1)} & =U(x, t) \\
U^{(2)} & =U(x, t)-i c \Delta t k_{F}^{*} U^{(1)} \\
& =\left(1-i c \Delta t k_{F}^{*}\right) U(x, t)
\end{aligned}
$$

Then:

$$
\begin{aligned}
U(x, t+\Delta t) & =U(x, t)-i c \frac{\Delta t}{2}\left[k_{F}^{*} U^{(1)}+k_{B}^{*} U^{(2)}\right] \\
& =U(x, t)-i c \frac{\Delta t}{2}\left[k_{F}^{*} U(x, t)+k_{B}^{*}\left(1-i c \Delta t k_{F}^{*}\right) U(x, t)\right] \\
& =\left[1-i c \frac{\Delta t}{2}\left(k_{F}^{*}+k_{B}^{*}\right)-\frac{(i c \Delta t)^{2}}{2}\left(k_{B}^{*} k_{F}^{*}\right)\right] U(x, t) \\
& =\left[1-i c \Delta t k^{*}+\frac{(-i c \Delta t)^{2}}{2}\left(\left(k^{*}\right)^{2}+\delta^{2}\right)\right] U(x, t)
\end{aligned}
$$


Using Eq. (13), it is clear that the numerical frequency for the MacCormack scheme is:

$$
\omega^{*}=\frac{-i}{\Delta t} \ln \left[1-i c \Delta t k^{*}+\frac{(-i c \Delta t)^{2}}{2}\left(\left(k^{*}\right)^{2}+\delta^{2}\right)\right]
$$

From this development, three contributions to the error are present in the MacCormack scheme: the error in dispersion from the spatial central difference, the error in dissipation from the one-sided spatial differences, and the error in both dispersion and dissipation from the time integration method itself.

\section{Higher Order Runge-Kutta Schemes for MacCormack-type Methods}

In order to investigate the effect of the time integration scheme on the dispersion and dissipation error, several high-order Runge-Kutta methods were constructed for MacCormack-type schemes. Eq. (10) gives the generic form of the Runge-Kutta schemes. For example, the two-stage, second-order accurate method given in Eq. (13) would have the coefficients given for the RK2 scheme.

It should be noted that, in order to achieve the desired spatial accuracy using a MacCormack-type scheme, the sum of the odd $\beta$ 's $(1,3,5)$ must add up to equal the sum of the even $\beta$ 's $(2,4,6)$. In this way, the central difference will be obtained from the sum of the forward and backward differences. Similar constraints are used for the odd time derivatives to insure that the one-sided differences sum properly.

Table 1 gives the coefficients for Runge-Kutta 2nd, 4th, and 6th order accurate methods, as well as the coefficients for Hu, et. al.'s optimized 4-6 Runge-Kutta scheme [19]. 


\begin{tabular}{|c|c|c|c|c|c|}
\hline & RK2 & RK4 & RK6 & RK4-6 Step1 & RK4-6 Step2 \\
\hline$\alpha_{2}$ & 1 & $1 / 2$ & $1 / 3$ & $1 / 2$ & .353323 \\
\hline$\alpha_{3}$ & 0 & $1 / 2$ & $1 / 3$ & $1 / 2$ & .353323 \\
\hline$\alpha_{4}$ & 0 & 1 & $3 / 8$ & 1 & .240823 \\
\hline$\alpha_{5}$ & 0 & 0 & $3 / 8$ & 0 & .240823 \\
\hline$\alpha_{6}$ & 0 & 0 & $64 / 21$ & 0 & .341148 \\
\hline$\beta_{1}$ & $1 / 2$ & $1 / 6$ & $-43 / 240$ & $1 / 6$ & -.766927 \\
\hline$\beta_{2}$ & $1 / 2$ & $1 / 3$ & $17 / 240$ & $1 / 3$ & -.519328 \\
\hline$\beta_{3}$ & 0 & $1 / 3$ & $33 / 80$ & $1 / 3$ & .147469 \\
\hline$\beta_{4}$ & 0 & $1 / 6$ & $2 / 5$ & $1 / 6$ & -.140084 \\
\hline$\beta_{5}$ & 0 & 0 & $4 / 15$ & 0 & 1.11946 \\
\hline$\beta_{6}$ & 0 & 0 & $7 / 240$ & 0 & 1.15941 \\
\hline$c_{1}$ & 1 & 1 & 1 & 1 & 1 \\
\hline$c_{2}$ & $1 / 2$ & $1 / 2$ & $1 / 2$ & $1 / 2$ & $1 / 2$ \\
\hline$c_{3}$ & 0 & $1 / 6$ & $1 / 6$ & $1 / 6$ & $1 / 6$ \\
\hline $\mathrm{C}_{4}$ & 0 & $1 / 24$ & $1 / 24$ & $1 / 24$ & $1 / 24$ \\
\hline$c_{5}$ & 0 & 0 & $1 / 120$ & 0 & 0.0162098 \\
\hline$c_{6}$ & 0 & 0 & $1 / 720$ & 0 & 0.00286365 \\
\hline
\end{tabular}

Table I: Coefficients for Runge-Kutta Time Stepping Schemes.

Following the development given for the 2-4 scheme, we find that a sixth order accurate 
Runge Kutta method gives this result:

$$
\omega^{*}=\frac{-i}{\Delta t} \ln \left[\begin{array}{l}
1-i c \Delta t k^{*}+\frac{(-i c \Delta t)^{2}}{2}\left(\left(k^{*}\right)^{2}+\delta^{2}\right)+ \\
\frac{(-i c \Delta t)^{3}}{6} k^{*}\left(\left(k^{*}\right)^{2}+\delta^{2}\right)+\frac{(-i c \Delta t)^{4}}{24}\left(\left(k^{*}\right)^{2}+\delta^{2}\right)^{2}+ \\
\frac{(-i c \Delta t)^{5}}{120} k^{*}\left(\left(k^{*}\right)^{2}+\delta^{2}\right)^{2}+\frac{(-i c \Delta t)^{6}}{720}\left(\left(k^{*}\right)^{2}+\delta^{2}\right)^{3}
\end{array}\right]
$$

Again, it is seen that the errors arise from the dispersion error in the central difference and the dissipation error in the one-sided differences.

From Eq. (23), it can be seen that the numerical frequency for a MacCormack-type scheme may be written generally as:

$$
\omega^{*}=\frac{-i}{\Delta t} \ln \left[1+\sum_{l=1}^{S / 2}\left\{\begin{array}{l}
c_{2 l-1}\left((-i c \Delta t)^{2 l-1} k^{*}\left[\left(k^{*}\right)^{2}+\delta^{2}\right]^{l-1}\right)+ \\
c_{2 l}\left((-i c \Delta t)^{2 l}\left[\left(k^{*}\right)^{2}+\delta^{2}\right]^{l}\right)
\end{array}\right\}\right]
$$

where $S$ is the number of stages in the Runge-Kutta scheme and the $c_{1}$ 's are the leading coefficients in the time integration, given in Table I.

\section{Improved Central Differences}

Various central differences were investigated during this work for use with MacCormacktype schemes. Fourth, sixth, and eighth order central differences were investigated, as well as the fourth-order accurate Dispersion Relation Preserving (DRP) scheme of Tam and Webb. 
The error in the dispersion relations was defined as:

$$
\text { Error }=k^{*} \Delta x-k \Delta x
$$

The dispersion errors are plotted as a function of wavenumber $\mathrm{k} \Delta \mathrm{x}$ in Figures 1 and 2 . It can be seen that higher orders of accuracy result in lower errors, as would be expected. However, lower order differences can be optimized as shown by Tam and Webb to provide better performance for a given level of acceptable error, illustrated in Figure 2.

\section{Improved One-Sided Differences}

\section{Higher-Order Accurate One-Sided Differences}

Using the basic methodology of the MacCormack method, improved accuracy can be attained by altering the one-sided differences to have higher accuracy while still adding up to a more accurate central difference. For a fourth-order accurate central difference, two schemes can be constructed:

The first scheme is the normal $4 / 2$ scheme, which is fourth order accurate in space and has second-order dissipation in each step. The next scheme is a $4 / 4$ scheme, which is fourth order accurate in space and has fourth-order dissipation in each step. This higher-order accuracy is achieved by adding one point to each of the one-sided differences, as shown in Table II.

Notice how both schemes add up to the identical 4 th order accurate central difference. The difference in the two schemes is in the leading error terms of the one-sided differences, which affects the inherent dissipation of the scheme. The effect of this change is to lower the inherent dissipation at a given wavenumber, as shown in Figure 3. This gives the 


\begin{tabular}{|c|c|c|c|c|c|c|c|}
\hline & $a_{-2}$ & $\underline{a_{-1}}$ & $a_{0}$ & $a_{1}$ & $a_{2}$ & $a_{3}$ & $a_{4}$ \\
\hline $2 / 2[1]$ & 0 & 0 & $\frac{-1}{\Delta x}$ & $\frac{1}{\Delta x}$ & 0 & 0 & 0 \\
\hline $4 / 2[2]$ & 0 & 0 & $\frac{-7}{6 \Delta x}$ & $\frac{8}{6 \Delta x}$ & $\frac{-1}{6 \Delta x}$ & 0 & 0 \\
\hline $6 / 2[17]$ & 0 & 0 & $\frac{-37}{30 \Delta x}$ & $\frac{45}{30 \Delta x}$ & $\frac{-9}{30 \Delta x}$ & $\frac{1}{30 \Delta x}$ & 0 \\
\hline $8 / 2$ & 0 & 0 & $\frac{-533}{420 \Delta x}$ & $\frac{672}{420 \Delta x}$ & $\frac{-168}{420 \Delta x}$ & $\frac{32}{420 \Delta x}$ & $\frac{-3}{420 \Delta x}$ \\
\hline $4 / 4$ & 0 & $\frac{-2}{6 \Delta x}$ & $\frac{-3}{6 \Delta x}$ & $\frac{6}{6 \Delta x}$ & $\frac{-1}{6 \Delta x}$ & 0 & 0 \\
\hline $6 / 4$ & 0 & $\frac{-9}{30 \Delta x}$ & $\frac{-19}{30 \Delta x}$ & $\frac{36}{30 \Delta x}$ & $\frac{-9}{30 \Delta x}$ & $\frac{1}{30 \Delta x}$ & 0 \\
\hline $\mathrm{DRP} / 4$ & 0 & $\frac{-.3766}{\Delta x}$ & $\frac{-.4968}{\Delta x}$ & $\frac{11651}{\Delta x}$ & $\frac{-.3334}{\Delta x}$ & $\frac{.04168}{\Delta x}$ & 0 \\
\hline DRP/opt & 0 & $\frac{-.30874}{\Delta x}$ & $\frac{-.6326}{\Delta x}$ & $\frac{12330}{\Delta x}$ & $\frac{-.3334}{\Delta x}$ & $\frac{.04168}{\Delta x}$ & 0 \\
\hline $8 / 4$ & 0 & $\frac{-120}{420 \Delta x}$ & $\frac{-293}{420 \Delta x}$ & $\frac{552}{420 \Delta x}$ & $\frac{-168}{420 \Delta x}$ & $\frac{32}{420 \Delta x}$ & $\frac{-3}{420 \Delta x}$ \\
\hline $6 / 6$ & $\frac{3}{60 \Delta x}$ & $\frac{-30}{60 \Delta x}$ & $\frac{-20}{60 \Delta x}$ & $\frac{60}{60 \Delta x}$ & $\frac{-15}{60 \Delta x}$ & $\frac{2}{60 \Delta x}$ & 0 \\
\hline $8 / 6$ & $\frac{18}{420 \Delta x}$ & $\frac{-192}{420 \Delta x}$ & $\frac{-185}{420 \Delta x}$ & $\frac{480}{420 \Delta x}$ & $\frac{-150}{420 \Delta x}$ & $\frac{32}{420 \Delta x}$ & $\frac{-3}{420 \Delta x}$ \\
\hline
\end{tabular}

Table II: Coefficients for MacCormack-Type Schemes

scheme a wider range of wavenumbers that it can accurately resolve, and thus requires less points per wavelength. 
Using this basic methodology, families of 6th and 8th order accurate schemes can be constructed. In these higher order schemes, it is possible to add another point to the onesided differences to attain 6 th order dissipation. These results are given in Table II.

\section{Optimization of the Split Operators}

The Tam and Webb optimization technique may be used on the one-sided differences to improve their performance at wavenumbers of interest. The procedure is as follows:

The central difference that the one-sided differences must add up to is fixed:

$$
\left.\frac{\partial U}{\partial x}\right|_{i} ^{C}=\left[\begin{array}{l}
A_{-3} U_{i-3}+A_{-2} U_{i-2}+A_{-1} U_{i-1}+A_{0} U_{i} \\
+A_{1} U_{i+1}+A_{2} U_{i+2}+A_{3} U_{i+3}
\end{array}\right]
$$

This, in turn, determines some of the coefficients of the one-sided difference:

$$
\left.\frac{\partial U}{\partial x}\right|_{i} ^{F}=2 A_{3} U_{i+3}+2 A_{2} U_{i+2}+a_{1} U_{i+1}+a_{0} U_{i}+a_{-1} U_{i-1}
$$

There are two conditions that are known for the three unknowns:

$$
\begin{aligned}
2 A_{3}+2 A_{2}+a_{1}+a_{0}+a_{-1} & =0 \\
a_{1}-a_{-1} & =2 A_{1}
\end{aligned}
$$

For the third condition, we can set a range to minimize the dissipation of the one-sided differences. For this work, the dissipation was minimized at 8 points per wavelength and higher. To do this, the following equation is minimized (following Tam and Webb's procedure):

$$
E=\left.\int_{-\frac{\pi}{4}}^{\frac{\pi}{4}} \operatorname{Re}\left[\sum_{j=-1}^{3} a_{j} e^{i j \kappa}\right]\right|^{2} d \kappa
$$


The condition that $E$ is a minimum is:

$$
\frac{\partial E}{\partial a_{-1}}=0
$$

This gives the condition required, and the coefficients are solved from the resulting linear system of equations. Figure 3 illustrates the effect of optimization on the inherent dissipation of the scheme. Notice that there is a price for this optimization: the lower wavenumbers (more points per wavelength) have slightly increased dissipation as compared to the original scheme.

The DRP schemes shown are given in Table II.

\section{Dissipative Ertors of the One-Sided Differences}

Figure 3 shows the dissipative errors in the one-sided differences for various MacCormack-type schemes. It can be seen that, in general, the dissipative performance of schemes with 2 nd order dissipation ( $4 / 2$ and $6 / 2$, for example) are similar. It can also be seen that the closer the split stencil is to the central difference stencil, the less dissipation the scheme will have $(6 / 6$, for example). It can be seen that, in general, higher-order accuracy reduces dissipation errors at a given wavenumber. The positive effect of the DRP-style optimization on the dissipation errors can also be seen in this figure.

\section{Performance of the Improved MacCormack-type schemes}

In this section, the total performance of each scheme will be quantified. In order to fairly compare the schemes, the errors in dissipation and dispersion per wavelength of travel will be shown as functions of wavenumber and time step. In this way, the effects of both the time and space discretization are shown. 
In these results, the following definitions are used:

$$
\begin{aligned}
& P P W=\frac{2 \pi}{k \Delta x} \\
& C F L=\frac{c \Delta t}{\Delta x} \\
& N S T=\frac{P P W}{C F L}
\end{aligned}
$$

Here, PPW is the number of spatial points per wavelength, CFL is the Courant number, and NST is the number of time steps per cycle.

From Eq. (11), we see that:

$$
\begin{aligned}
U(x, t+N S T \Delta t) & =U(x, t) e^{-i(\omega N S T \Delta t)} \\
& =U(x, t)\left(e^{-i(\omega \Delta t)}\right)^{N S T}
\end{aligned}
$$

Since the problem is periodic,

$$
\begin{gathered}
U(x, t+N S T \Delta t)=U(x, t) \\
\left(e^{-i(\omega \Delta t)}\right)^{N S T}=1+0 i
\end{gathered}
$$

and we can define the numerical solution to be:

$$
\left(e^{-i\left(\omega^{\cdot} \Delta t\right)}\right)^{N S T}=A+i D
$$

Here, $\mathrm{A}$ is the amplitude per wavelength traveled and $\mathrm{D}$ is the dispersion error per wavelength traveled.

Figure 4 shows the amplitude and dispersion per wavelength traveled for the previously published MacCormack-type schemes. It is seen that there is a large amount of dissipation inherent in these schemes, acting over a large part of the wavenumber range. Also, the dispersion plots emphasize the low order of accuracy in time. 
Figure 5 illustrates the effect that varying the time integration method has on the MacCormack-type schemes. In Figure 5, the 6/4 one-sided differences are used, and various time integration methods are employed. Notice how the variation of the errors with increasing time step is greatly reduced with the 46 and 6 stage time integration methods. Also, the optimized 46 time integration method is very nearly as accurate as the 6-stage, 6 th order method while requiring less work.

Figure 6 compares the $2-4 / 2$ method with the best of the two newer methods: the $46-6 / 4$ method and the 46-DRP/optimized method. Notice that using the DRP method can extend the usable wavenumber range for a given amount of error significantly without requiring any additional calculations. Also, the stability range of the newer schemes are much greater than that of the older MacCormack-type schemes, while retaining very good accuracy.

Figures 7-9 shows the final results of this work: the actual wave propagation.

In Figure 7 , a wave of 8 points per wavelength $(\mathrm{k} \Delta \mathrm{x}=.785)$ is propagated for 400 time steps at a CFL of 0.5 (25 wavelengths of travel). Results are shown for the original 2-4/2 scheme, the 46-6/4 scheme, and the optimized 46-DRP/opt scheme. The low CFL number is chosen due to the low stability limits of the $2-4 / 2$ scheme.

It can be seen that the $2-4 / 2$ scheme is the most dissipative, followed by the $46-6 / 4$ scheme and the 46-DRP/opt scheme. Referring to Figure 3, the magnitudes of the dissipation for these schemes fall in the same sequence..

The dispersive error is illustrated in Figure 1. The fourth-order central difference has the highest dispersive error, with the numerical wave trailing the actual wave. The sixth-order central difference and the DRP central difference have about the same amount of dispersion, with the sixth order wave trailing the actual wave, and the DRP wave leading. Again, Figure 1 illustrates this effect. 
Moving on to higher CFL, Figure 8 shows the same problem with a CFL of 1.25 ( 8 points per wavelength, 25 wavelengths of travel, 160 time steps). The 2-4/2 scheme was unstable at this larger time step, so only the results from the other two schemes are shown. It can be seen that the results are almost identical to the previous calculation, which illustrates the large time steps possible with the optimized 46 time stepping scheme.

Figure 9 shows the results for 6 points per wavelength $(\mathrm{k} \Delta \mathrm{x}=1.05)$ after 33.33 wavelengths of travel ( $\mathrm{CFL}=1.25,160$ time steps). The improvement in both dispersion and dissipation of the DRP scheme can be seen compared to the 46-6/4 scheme, illustrating the benefits of optimization for this severe problem.

\section{Conclusions}

In this work, the dispersive and dissipative characteristics of the existing MacCormack-type scheme were investigated, and several ways were found to improve the accuracy of this type of scheme. The accuracy and stability of these schemes were greatly enhanced without much additional calculations being needed.

The MacCormack-type schemes are of great interest due to their ease of programming and use, and inherent numerical dissipation. This work shows that this type of scheme can be optimized to perform very well. 


\section{References}

1. R. W. MacCormack, 'Lecture Notes in Physics', Vol. 8, p. 151, Springer-Verlag, New York/Berlin, 1971.

2. D. Gottleib and E. Turkel, Math. of Comp., 30,703 (1976).

3. A. Bayliss and L. Maestrello, AIAA J., 19, 835 (1981).

4. L. Maestrello, A. Bayliss, and E. Turkel, J. Sound and Vib., 74, 281 (1981).

5. A. Bayliss and L. Maestrello, J. Sound and Vib., 86, 395 (1982).

6. L. Maestrello and A. Bayliss, AIAA J., 20, 1539 (1982).

7. A. Bayliss, L. Maestrello, and E. Turkel, J. Sound and Vib., 107, 167 (1986).

8. R. R. Mankbadi, M. E. Hayder, and L. A. Povinelli, AIAA J., 32, 897 (1994).

9. R. R. Mankbadi, S.-H. Shih, R. Hixon, and L. A. Povinelli, 'Direct Computation of Acoustic and Flow Field of a Supersonic Jet Using Large-Eddy Simulation', AIAA Paper 95-0680, Reno, NV, Jan. 1995.

10. S.-H. Shih, R. Hixon, and R. R. Mankbadi, 'A Zonal Approach for Prediction of Jet Noise', CEAS/AIAA Paper 95-144, Munich, Germany, June 1995.

11. R. R. Mankbadi, R. Hixon, S.-H. Shih, and L. A. Povinelli, 'On the Use of Linearized Euler Equations in the Prediction of Jet Noise', AIAA Paper 95-0505, Reno, NV, Jan. 1995.

12. R. Hixon, S.-H. Shih, and R. R. Mankbadi, 'Direct Prediction of the ThreeDimensional Acoustic Field of a Supersonic Jet Using Linearized Euler Equations', CEAS/AIAA-95-116, June 1995. 
13. R. Hixon, S.-H. Shih, and R. R. Mankbadi, 'Numerical Simulation of the Effect of Heating on Jet Noise', FED-Vol.. 219, Computational Aeroacoustics, ASME 1995, p. 89-94.

14. R. Hixon, S.-H. Shih, and R. R. Mankbadi, AlAA J., 33, 2006 (1995).

15. R. Hixon, S.-H. Shih, and R. R. Mankbadi, 'Effect of Inflow Disturbance on Linearized Euler Equation Prediction of Jet Noise', AIAA Paper 96-0752, Jan. 1996.

16. L. N. Sankar, N. N. Reddy, and N. Hariharan, 'A Comparative Study of Numerical Schemes for Aero-Acoustic Applications', FED-Vol. 147, Computational Aeroand Hydro-Acoustics, ASME 1993, p. 35-40.

17. A. Bayliss, P. Parikh, L. Maestrello, and E. Turkel, 'Fourth Order Scheme for the Unsteady Compressible Navier-Stokes Equation', ICASE Report 85-44, Oct. 1985.

18. C. K. W. Tam and J. C. Webb, J. Comp. Physics, 107, 262 (1993).

19. F. Q. Hu, M. Y. Hussaini, and J. Manthey, 'Low-Dissipation and -Dispersion Runge-Kutta Schemes for Computational Acoustics', ICASE Report 94-102, Dec. 1994. 


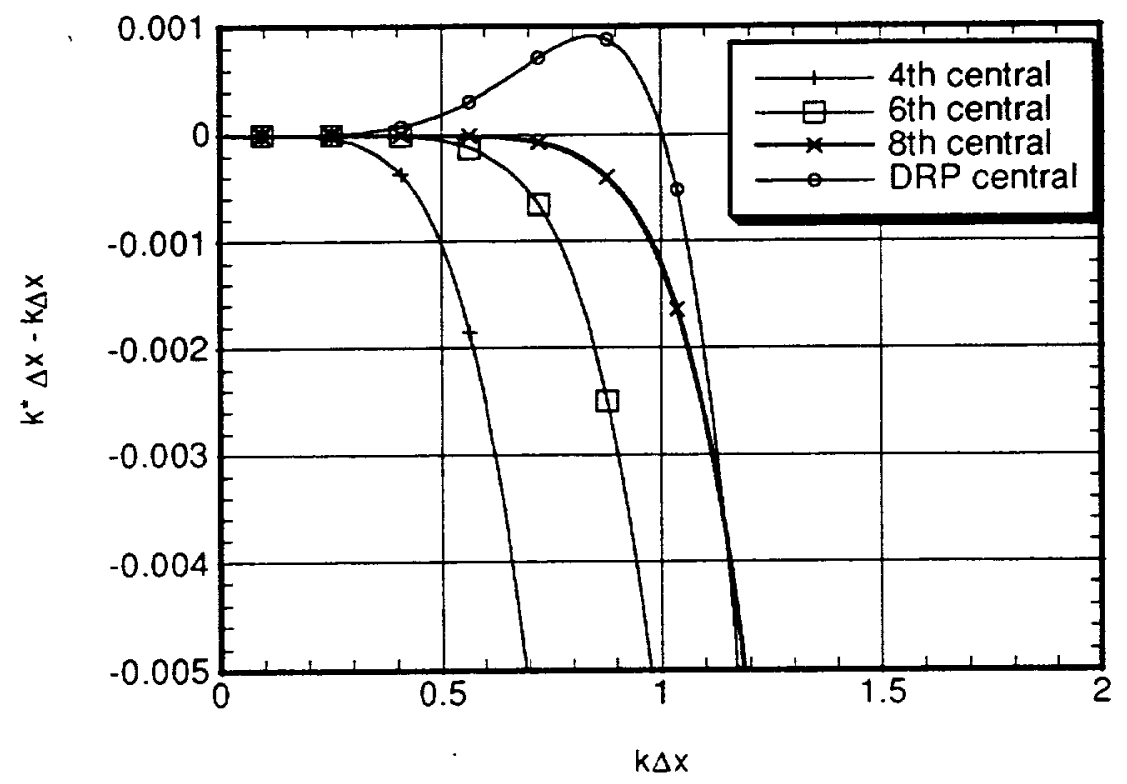

Figure 1

Effect of Central Difference on the Onset of Dispersion Error. 


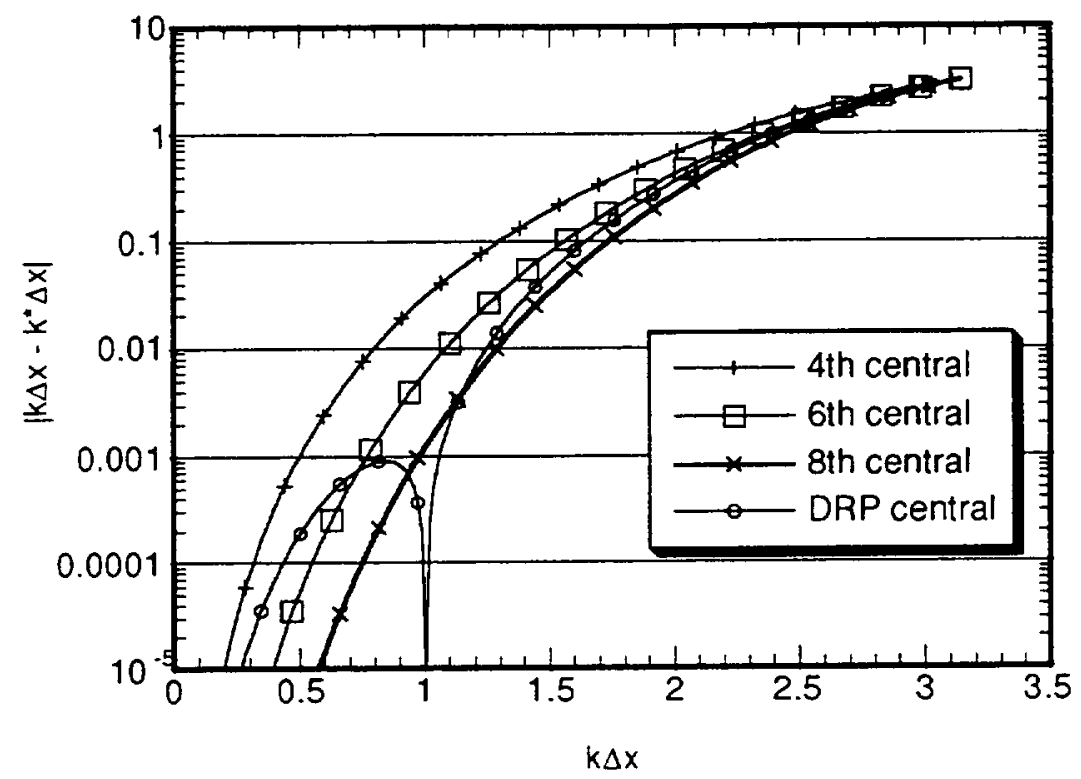

Figure 2

Effect of Central Difference on the Magnitude of the

Dissipation Error. 


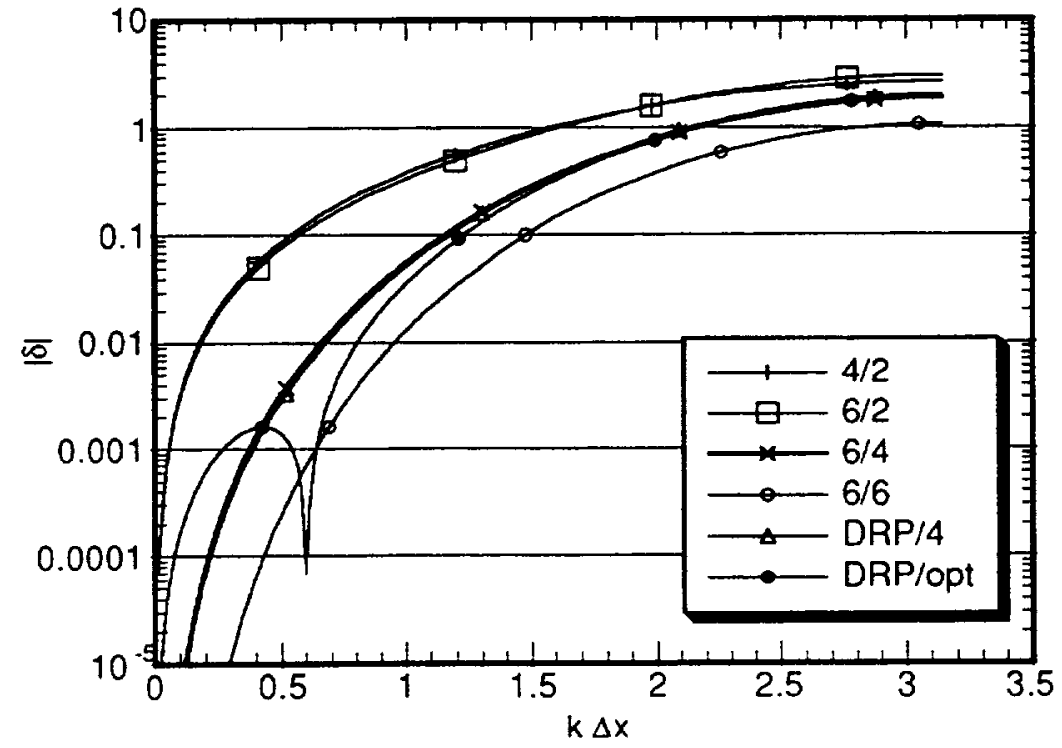

Figure 3

Effect of One-Sided Differences on the Magnitude of the Dissipation Error. 
Amplitude

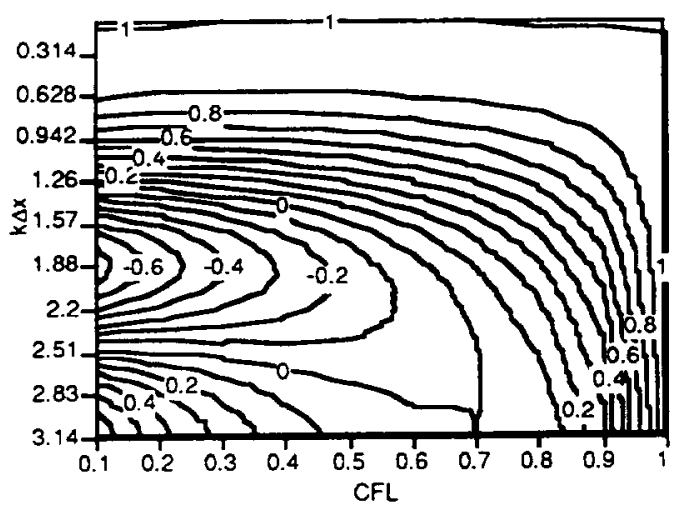

\section{Dispersion error}

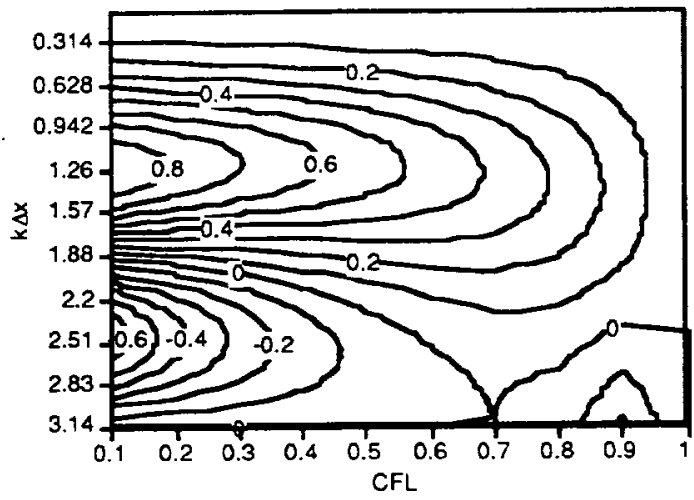

a) $2-2 / 2$ scheme
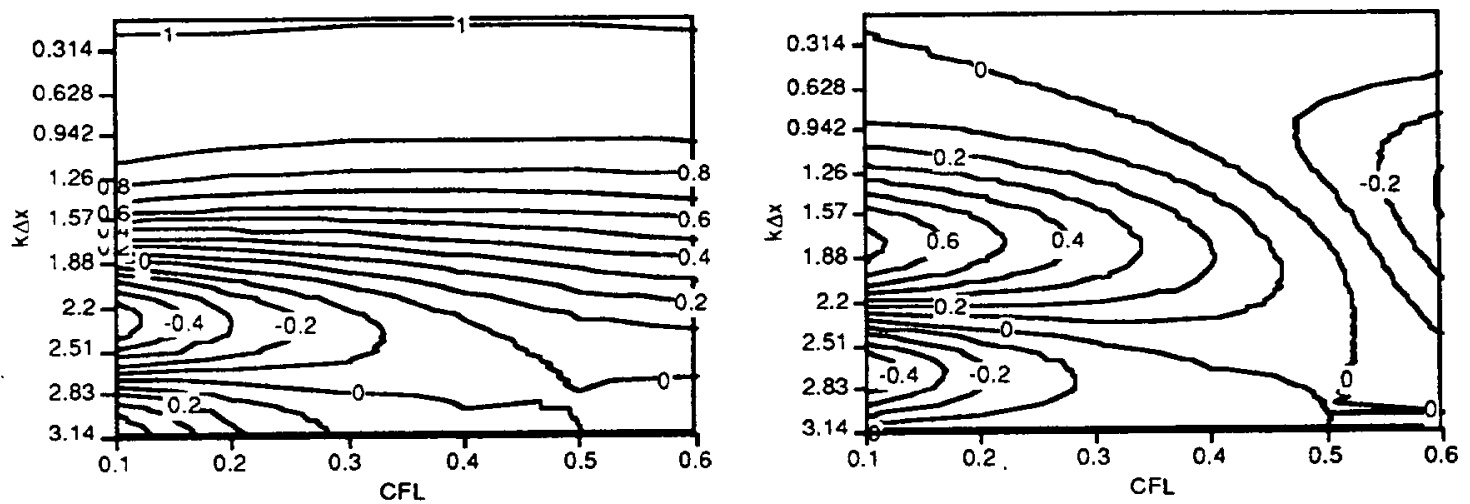

b) 2-4/2 scheme
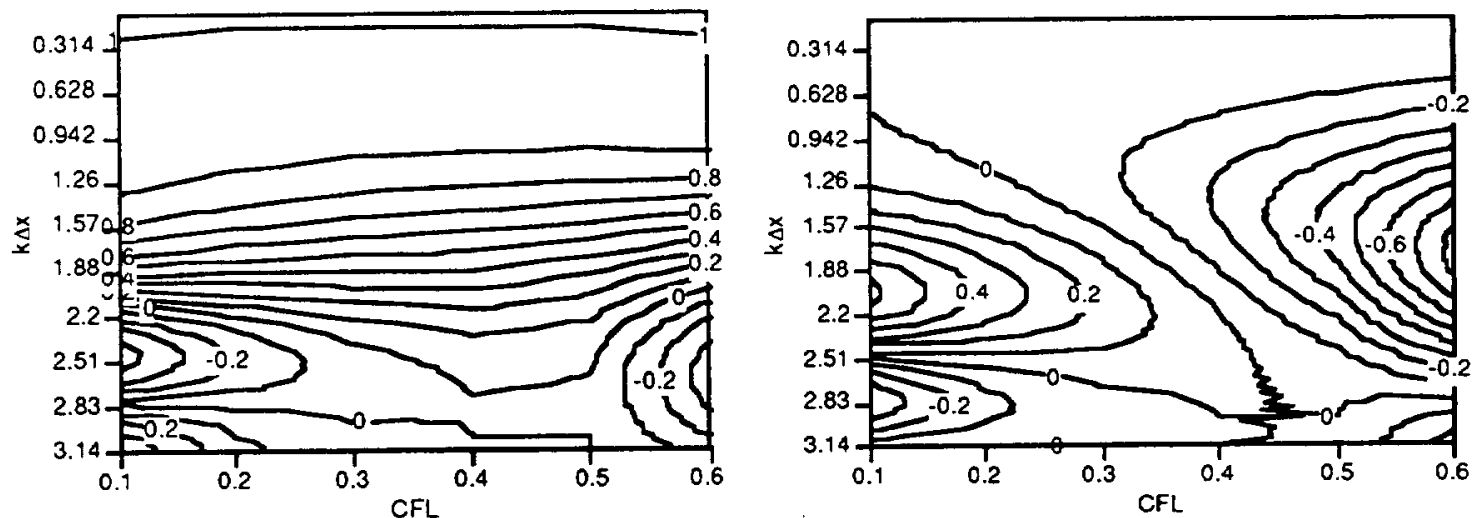

c) $2-6 / 2$ scheme

Figure 4

Performance of Previous MacCormack-Type Schemes. 
Amplitude

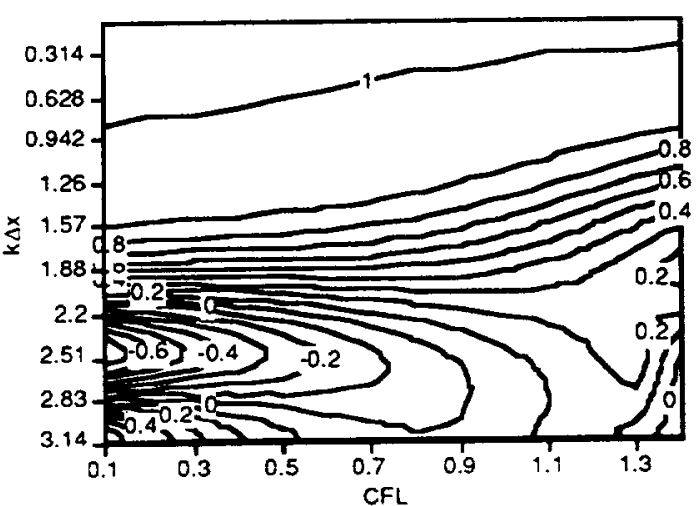

Dispersion error

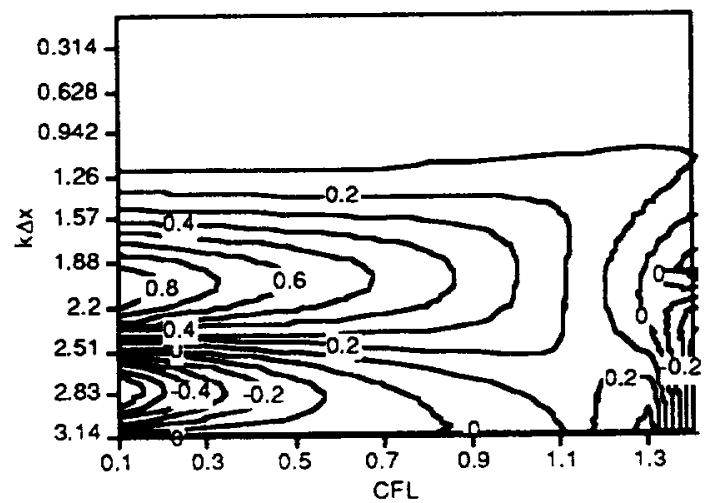

a) 4-6/4 scheme
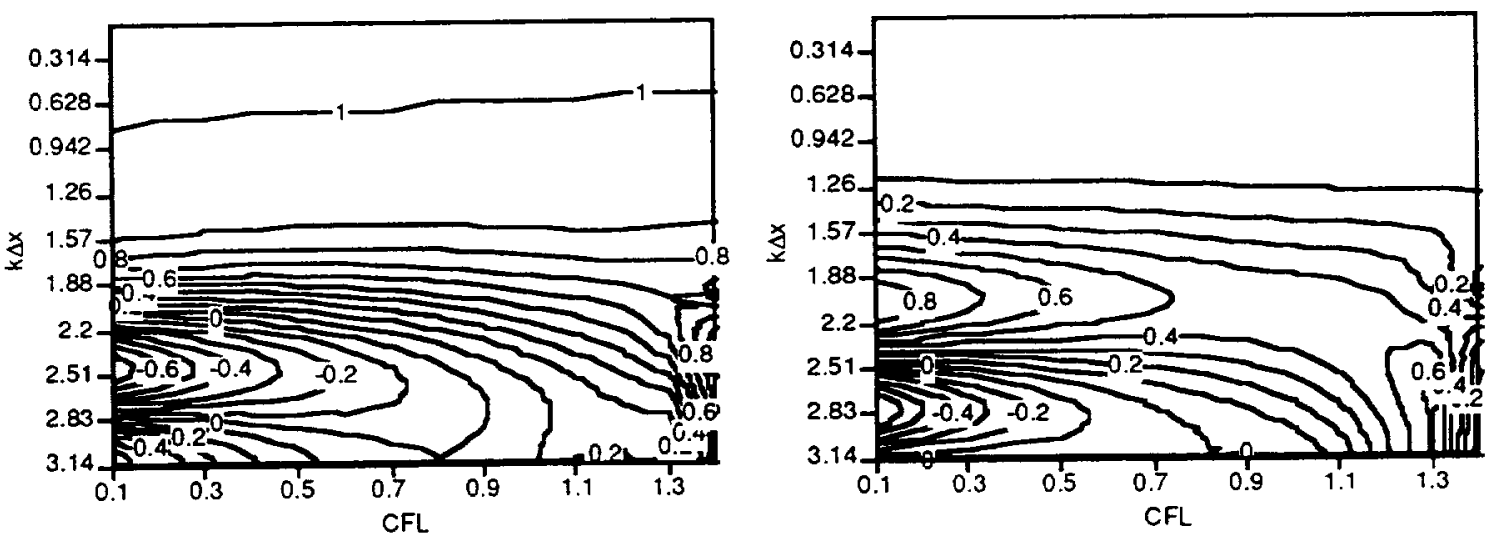

b) $46-6 / 4$ scheme
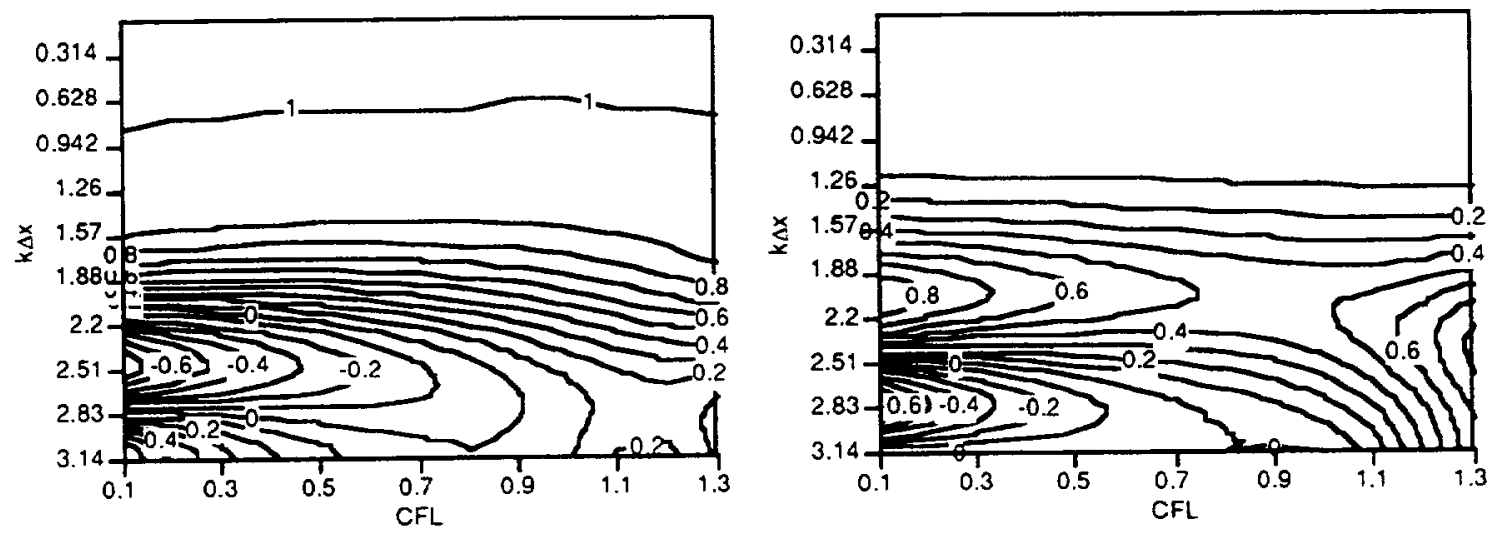

c) 6-6/4 scheme

\section{Figure 5}

Effect of Time Integration Method on Accuracy. 
Amplitude

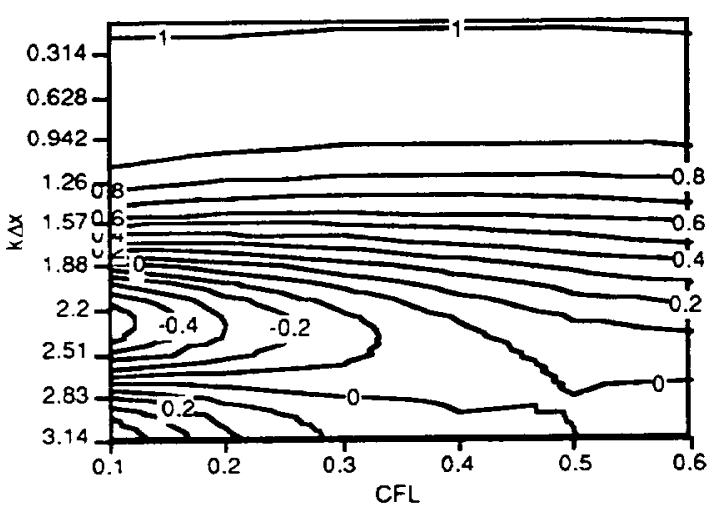

a) 2-4/2 scheme

\section{Dispersion error}

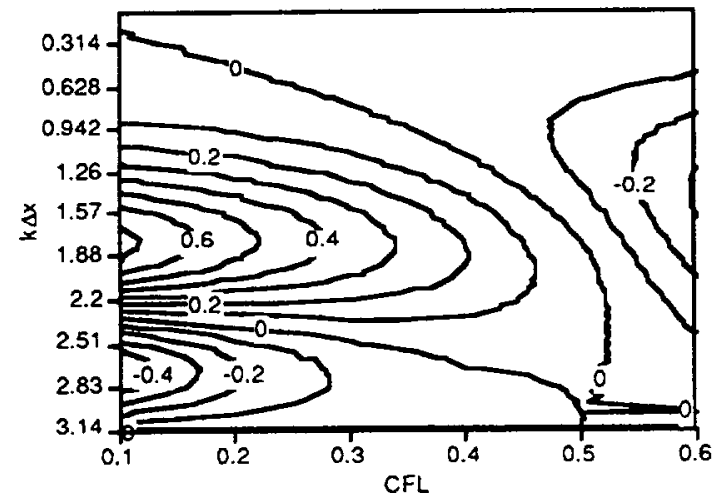




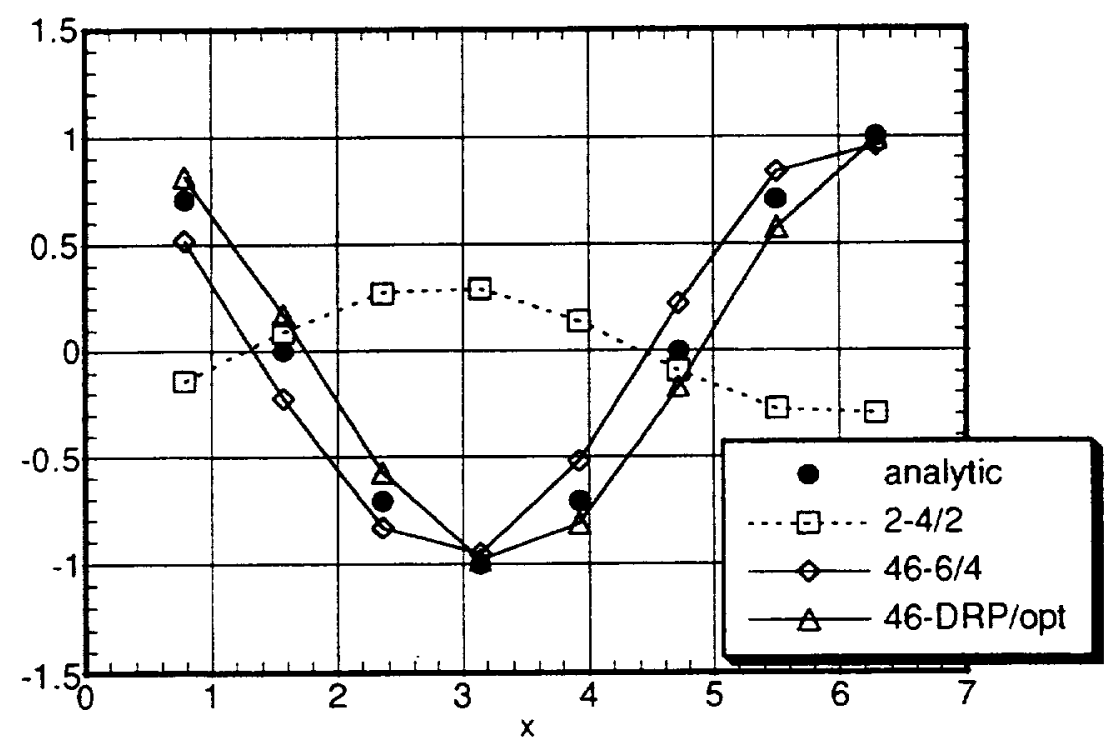

Figure 7

Comparison of results obtained by MacCormack-type methods (8 ppw, CFL $=0.5,25$ wavelengths of travel) 


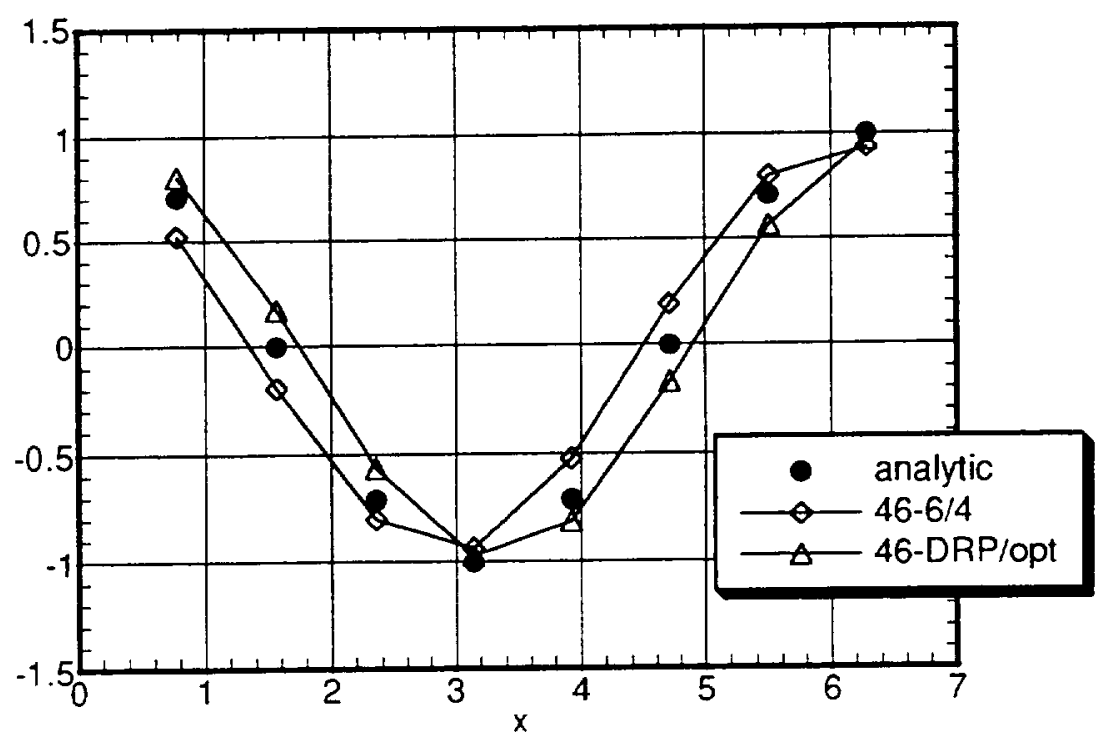

Figure 8

Comparison of results obtained by MacCormack-type methods ( 8 ppw, CFL $=1.25,25$ wavelengths of travel) 


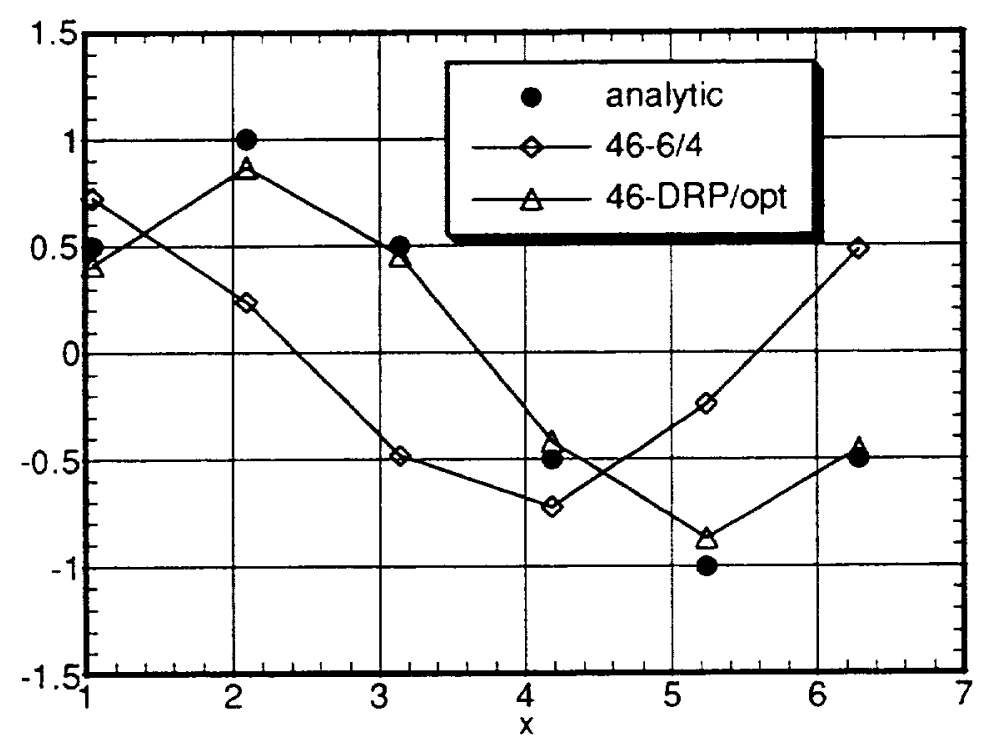

Figure 9

Comparison of results obtained by MacCormack-type methods (6 ppw, CFL $=1.25,33.3$ wavelengths of travel) 
Public reporting burden lor this collection of Intormation is estimated to average 1 hour per response, including the time lor revieming instructions, searching existing data sources,

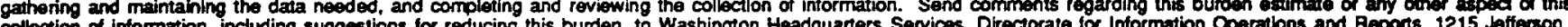
Davis Highway. Suite 1204. Arlington, VA 22202-4302, and to the Otfice of Management and Budget, Paperwork Reduction Projed (0704-0183), Washington, DC 20503.

\begin{tabular}{|l|l|l}
\hline 1. AGENCY USE ONLY (Leave blank) & $\begin{array}{c}\text { 2. REPORT DATE } \\
\text { December } 1996\end{array}$ & $\begin{array}{c}\text { 3. REPORT TYPE AND DATES COVERED } \\
\text { Contractor Report }\end{array}$
\end{tabular}

\section{TITLE AND SUBTIRE}

On Increasing the Accuracy of MacCormack Schemes for Aeroacoustic Applications

6. AUTHOR(S)

R. Hixon
5. FUNDING NUMBEAS

WU-505-90-5K

NCC3-483

8. PERFORMING ORGANIZATION REPORT NUMBER

E-10599

10. SPONSORINGMONITORING AGENCY REPORT NUMBER

NASA CR-202311

ICOMP-96-11

11. SUPPLEMENTARY NOTES

ICOMP Program Director, Louis A. Povinelli, organization code 2600, (216) 433-5818.

12a. DISTRIBUTIONAVAILABILTY STATEMENT

12b. DISTRIBUTION CODE

Unclassified - Unlimited

Subject Category 64

This publication is available from the NASA Center for AeroSpace Information, (301) 621-0390.

13. ABSTRACT (Maximum 200 words)

Due to their inherent dissipation and stability, the MacCormack scheme and its variants have been widely used in the computation of unsteady flow and acoustic problems. However, these schemes require many points per wavelength in order to propagate waves with a reasonable amount of accuracy. In this work, the linear wave propagation characteristics of MacCormack-type schemes are investigated, and methods for greatly improving their performance are described and demonstrated.

\section{SUBJECT TERMS}

MacCormack scheme; Time accuracy; Computational aeroacoustics

15. NUMBER OF PAGES

30

16. PRICE CODE

$\mathrm{A03}$

\begin{tabular}{|c|c|}
\hline $\begin{array}{c}\text { 17. SECURTY CLASSIFICATION } \\
\text { OF REPORT } \\
\text { Unclassified }\end{array}$ & $\begin{array}{c}\text { 18. SECURTY CLASSIFICATION } \\
\text { OF THIS PAGE } \\
\text { Unclassified }\end{array}$ \\
\hline
\end{tabular}

NSN 7540-01-280-5500
19. SECURITY CLASSIFICATION OF ABSTRACT

Unclassified
20. LIMITATION OF ABSTRACT 